\title{
Fascioliasis: una causa infrecuente de obstrucción de vía biliar. Reporte de un caso
}

\author{
Amada Belén Geldres Molina ${ }^{1} \odot \cdot$ Juan Luis Romero García ${ }^{2} \odot$ • Laura Isabel García Honores ${ }^{2}$ \\ Christian Eduardo Alcántara Figueroa ${ }^{3}$ \\ ${ }^{1}$ Estudiante de la Facultad de Medicina de la Universidad Privada Antenor Orrego. \\ ${ }^{2}$ Estudiante de la Facultad de Medicina Humana de la Universidad Privada Antenor Orrego. \\ ${ }^{3}$ Docente de la Universidad Privada Antenor Orrego. Médico Gastroenterólogo del Hospital Belén de Trujillo. \\ Perú
}

Acta Gastroenterol Latinoam 2021;51(3):347-352

Recibido: 13/01/2021 / Aceptado: 04/06/2021 / Publicado online: 27/9/2021 / bttps://doi.org/10.52787/werx8637

\section{Resumen}

La fascioliasis hepática es una enfermedad parasitaria producida por el trematodo Fasciola hepatica. En la fase crónica de la enfermedad, la forma adulta del parásito adopta los conductos biliares como el ambiente ideal para desarrollarse, causando la dilatación de estos y simulando un proceso ictérico obstructivo. Se presenta el caso de una mujer de 48 años, que ingresó por ictericia, coluria y pérdida de peso. Luego de que se realizaran los estudios complementarios correspondientes, se llevó a cabo una colangiografía retrógrada endoscópica (CPRE) en la que se extrajo el parásito adulto de Fasciola hepatica de la vía biliar. Posteriormente, se inició tratamiento antihelmintico con triclabendazol, lo que resultó en una evolución favorable y en la resolución del cuadro.

Palabras claves. Fasciola hepatica, colestasis, colangiopancreatografía retrógrada endoscópica.

Correspondencia: Amada Belén Geldres Molina

Correo electrónico: ageldresm1@upao.edu.pe

\section{Fascioliasis: an Uncommon Cause of Bile Duct Obstruction. A Case Report}

\section{Summary}

Hepatic fascioliasis is a parasitic disease caused by the fluke Fasciola hepatica. In the chronic phase of the disease, the adult form of the parasite adopts the bile ducts as the ideal environment to develop, causing them to dilate and simulating an obstructive jaundice process. The case of a 48-year-old woman is presented, who was admitted for jaundice, choluria and weight loss. After the corresponding complementary studies had been carried out, an endoscopic retrograde cholangiography (ERCP) was performed and an adult Fasciola hepatica parasite was extracted from the biliary tract. Subsequently, anthelmintic treatment with triclabendazole was started, resulting in a favorable evolution and resolution of the condition.

Keywords. Fasciola hepatica, cholestasis, endoscopic retrograde cholangiopancreatography.

\section{Abreviaturas \\ CPRE: Colangiografía retrógrada endoscópica. \\ ERCP: Endoscopic retrograde cholangiography.}

\section{Introducción}

La fascioliasis hepática es una enfermedad parasitaria distribuida mundialmente, causada por los trematodos 
Fasciola hepatica y Fasciola gigantia, siendo el primero el de mayor distribución global. Estos parásitos platelmintos tienen una predilección por el árbol biliar intrahepático o extrahepático. Los factores que contribuyen a la distribución geográfica dependen del hospedador intermediario -Lymnaea $s p p-\mathrm{y}$ de algunas condiciones, tales como las conductas alimentarias, los animales infectados, la falta de educación, la pobreza y el clima. Los Fasciola hepatica y Fasciola gigantica se notifican principalmente en zonas templadas y en regiones tropicales respectivamente. A nivel mundial, la zona andina de América del Sur es la zona más afectada por Fasciola hepatica, por lo que, en Cajamarca, Perú, es una parasitosis endémica. Las tasas de prevalencia en este lugar oscilan entre el $6 \%$ y el $68 \% .^{1}$ Actualmente, la fascioliasis no solo se reporta en las zonas rurales, sino que también hay casos en ciudades industrializadas, lo que se asocia con hábitos higiénicodietéticos, como no lavarse las manos y el consumo de vegetales contaminados. También influyen en este sentido las nuevas técnicas de diagnóstico. La infección por Fasciola hepatica y sus complicaciones son más frecuentes en el género femenino. En el Perú, el $71 \%$ de las regiones del territorio andino estarían afectadas por esta zoonosis. ${ }^{2}$

Es infrecuente pensar en Fasciola hepatica como una etiología de obstrucción biliar, por ello es importante el reporte de este caso.

\section{Caso clínico}

Se presenta el caso de una paciente de sexo femenino de 48 años, agricultora procedente de Cuzco, Perú, sin comorbilidades ni antecedentes clínicos, ginecológicos o quirúrgicos de importancia.

La paciente refiere que desde hace nueve meses presenta dolor abdominal en hipocondrio derecho, que inicia de forma insidiosa y cursa progresivamente de leve a moderada intensidad. Este dolor es opresivo y se irradia a epigastrio, dura aproximadamente dos horas y se acompaña de náuseas y vómitos, sensación de alza térmica, malestar general y cefalea, así como de episodios de deposiciones líquidas de regular volumen, sin moco ni sangre, que se repiten de forma intermitente.

Finalmente, la paciente acude a nuestra institución tras notar una coloración amarillenta en la piel, dolor abdominal, coluria y otros síntomas asociados, como debilidad, cansancio y una pérdida de peso de $8 \mathrm{~kg}$ en los últimos tres meses.

$\mathrm{Al}$ examen físico la paciente se encuentra en mal estado general, desnutrida y deshidratada. Además, se muestra ictérica $(++/+++)$, sin edemas y hemodinámicamente estable. En la exploración abdominal se palpa un abdo- men blando depresible, con dolor leve en hipocondrio derecho y epigastrio. También se palpa el borde hepático $4 \mathrm{~cm}$ por debajo del reborde costal, siendo doloroso a la palpación profunda, con signo de Murphy negativo (-). No se palpan masas. Se solicitan exámenes de laboratorio, los cuales revelan anemia moderada normocítica normocrómica, leucocitosis con eosinofilia al 60\% y hepatograma con patrón de alteración mixto. En el estudio coproparasitológico seriado se observan huevos anembrionados de Fasciola hepatica (Figura 1).

Figura 1. Se observan huevos anembrionados de Fasciola hepatica en heces del portador.

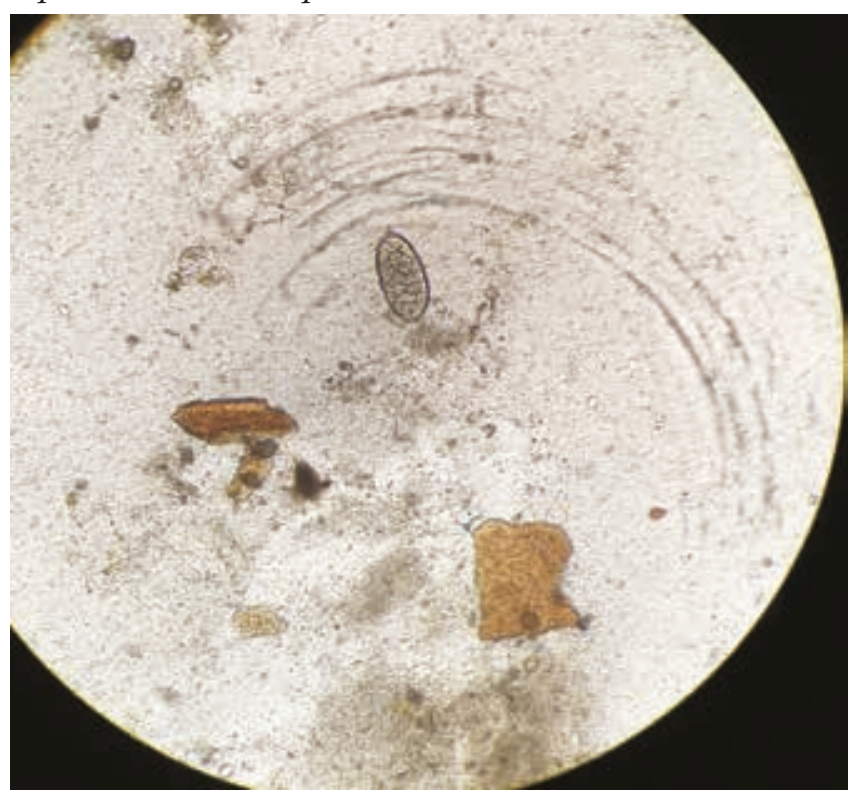

También fueron realizados estudios de imágenes. En la ecografía se reporta lóbulo hepático derecho de $180 \mathrm{~mm}$, parénquima heterogéneo y ecogenicidad aumentada, vesícula de $4,5 \mathrm{~cm}$, su pared de $5,3 \mathrm{~mm}$, el barro biliar ocupa $40 \%$ de la luz y colédoco de $10 \mathrm{~mm}$. En la tomografía espiral multicorte de abdomen completo con contraste, se describe: parénquima heterogéneo, presencia de imagen nodular en segmento II y una moderada dilatación de las vías biliares intra y extrahepáticas (Figuras 2 y 3 ).

La conclusión de este examen fue una hepatomegalia con signos de moderada esteatosis y dilatación de las vías biliares intra y extrahepáticas, con signos de proceso obstructivo en la ampolla de Vater.

En pacientes que cursan un síndrome coledociano, con eosinofilia marcada y que tienen antecedente de procedencia de una zona endémica, es importante realizar el diagnóstico diferencial con otras causas de infección para- 
Figuras 2 y 3. TAC abdominal contrastada

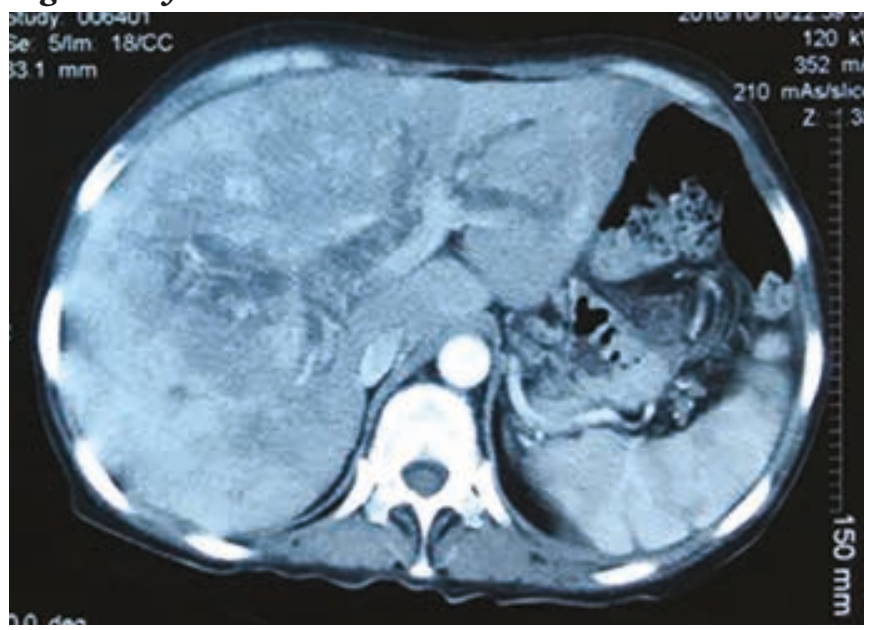

Imagen nodular en segmento || que realza contraste. Vía biliar dilatada.

sitaria que pueden ocasionar una obstrucción de la vía biliar, teniendo en cuenta los antecedentes antes mencionados (procedencia de zona endémica, una clínica subaguda o crónica y una eosinofilia marcada, entre otras). Entre las otras infecciones parasitarias, podemos mencionar a Opisthorchis viverrini, Fasciola gigantica, Ascaris lumbricoides (presente en climas cálidos, se identifica como una imagen cilíndrica móvil) y Clonorchis sinensis, ${ }^{3}$ siendo los dos últimos parásitos endémicos en el Perú.

También se ha descrito que la clínica de la fase crónica de la enfermedad puede manifestarse atípicamente, llegando a simular un colangiocarcinoma, caracterizado por ictericia indolora y disminución de peso. ${ }^{4,5}$

Cabe mencionar que, en caso de coledocolitiasis, el dolor abdominal tendría una presentación aguda y estaría asociado a la ingesta de alimentos.

Por todo lo dicho, se le realizó a la paciente una colangiografía retrógrada endoscópica (CPRE), ante la sospecha de obstrucción biliar parasitaria por fascioliasis crónica, con el objetivo de prevenir la aparición de una colangitis, una pancreatitis o una colecistitis. La CPRE se llevó a cabo en la sala de procedimientos endoscópicos del servicio de Gastroenterología, a través de sedación endovenosa con propofol. Con duodenoscopio Olympus TJF-Q180V, se visualizó la ampolla de Vater de aspecto abombado, y se intentó canular con papilotomo en tres oportunidades, sin éxito, por lo que se realizó precorte tipo fistulotomía utilizando cuchillo de precorte. Se accedió a la vía biliar, se opacificó el colédoco en $14 \mathrm{~mm}$ y se apreciaron en el colangiograma estructuras nodulares móviles. Se amplió el corte hasta el pliegue marginal. Se apreció salida de helmintos compatibles con Fasciola hepatica. Se procedió a la extracción de los parásitos con

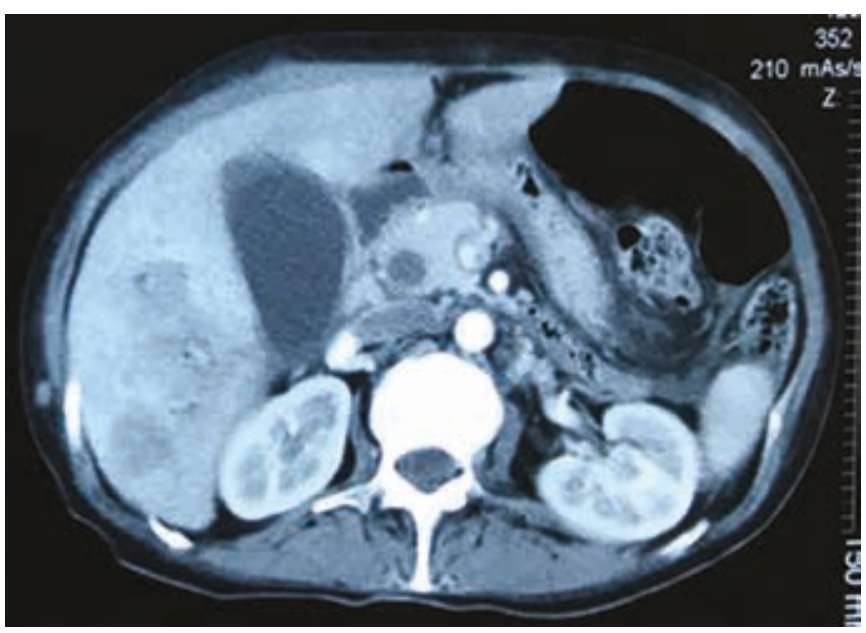

canastilla de Dormia. Se verificó colangiograma limpio. Se finalizó el procedimiento sin complicaciones. (Figuras $4,5$ y 6$)$.

Figuras 4 y 5. Durante la CPRE, se observó con el duodenoscopio la Fasciola hepatica adulta.

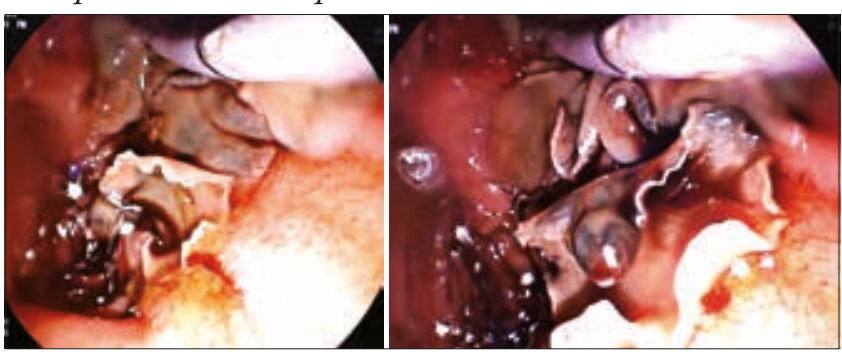

Figuras 6. Hallazgo de dos especimenes adultos de Fasciola hepatica extraidos.

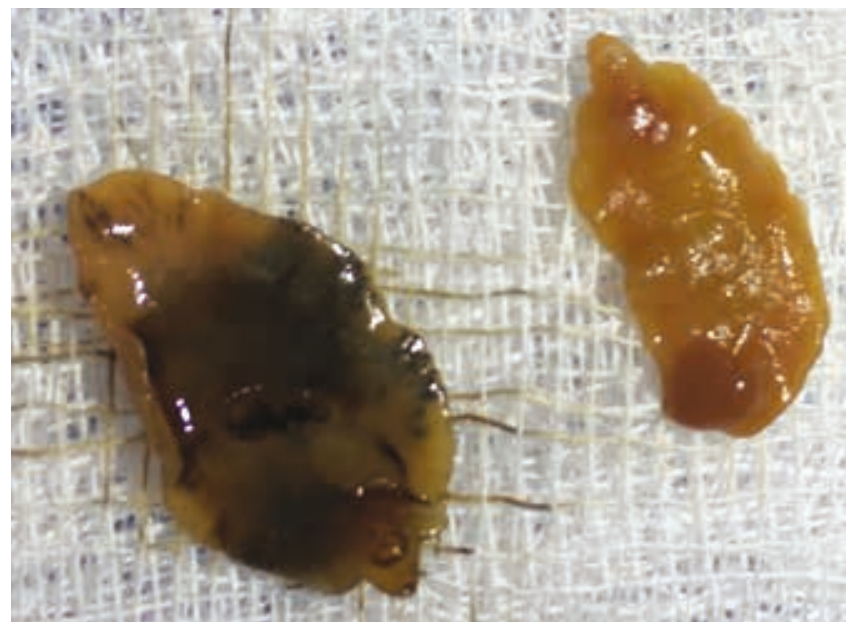




\section{Tratamiento}

La paciente recibió una sola dosis de triclabendazol $10 \mathrm{mg} / \mathrm{kg}$ vía oral.

\section{Evolución y seguimiento}

La paciente fue evaluada seis meses después de haber recibido el tratamiento farmacológico; se mostraba asintomática, sin anemia y con un aumento de peso de $6 \mathrm{~kg}$.

\section{Discusión}

La Fasciola hepatica es el agente causal de la fascioliasis. El huésped intermediario de este trematodo es el caracol de agua dulce de la especie Lymnae, mientras que el huésped definitivo son el ganado vacuno, el ovino y los seres humanos, quienes se infectan al consumir metacercarias que se enquistaron sobre plantas acuáticas, como el berro o la lechuga. Al llegar al intestino delgado del huésped definitivo, se desenquistan y atraviesan la pared intestinal, de allí pasan al hígado a través del peritoneo y, posteriormente, penetran en la cápsula de Glisson, para alcanzar y ubicarse en la vesícula y en los conductos biliares, donde dan origen a los adultos que producirán huevos, los cuales abandonarán al huésped para iniciar un nuevo el ciclo. En algunas ocasiones, el parásito llega a viajar a través de la sangre, por la cual es transportado a otras localizaciones. ${ }^{3}$

La sintomatología, aunque es variable, tiene dos etapas descritas. La primera etapa es la de infección aguda, que corresponde a la invasión hepática y dura hasta cuatro meses. El paciente suele ser asintomático o caracterizarse por la tríada de dolor en hipocondrio derecho o epigastralgia, fiebre continua y hepatomegalia dolorosa. Puede acompañarse de manifestaciones sistémicas, de las cuales no se conoce su mecanismo fisiopatológico, pero se plantea que pueden deberse a depósitos de inmunocomplejos circulantes; estas manifestaciones pueden ser: artralgias, mialgias, astenia, anorexia, adelgazamiento, síntomas de alergia -como prurito y urticaria-, sintomatología torácica -como derrame pleural-, tos seca con infiltrado pulmonar, síntomas cardíacos -como miocarditis o pericarditis- o manifestaciones neurológicas - como cefalea, meningoencefalitis y crisis convulsivas-. La segunda etapa es la etapa crónica, en la cual se encuentran la mayoría de los pacientes al momento del diagnóstico. Esta etapa continúa a las manifestaciones agudas; el paciente puede permanecer asintomático o, en caso contrario, presentar una clínica de obstrucción biliar, que puede ser parcial y cuyo síntoma característico es el dolor crónico en el hipocondrio derecho, que simula un cuadro de colecistitis crónica reagudizada. En cambio, si la obstrucción es completa, se presenta ictericia y se requie- re CPRE de emergencia. ${ }^{4,5}$ En algunos casos también hay manifestaciones de diseminación parasitaria extrahepática. Las manifestaciones extrahepáticas se dan por la migración ectópica del parásito, lo que puede producir infiltrado de eosinófilos y mononucleares y provocar daño tisular severo.6 El cuadro clínico del caso reportado es un cuadro de evolución crónica, similar a otro caso reportado anteriormente, de una mujer de 43 años con dolor abdominal recurrente, que tenía una historia de cinco años de colelitiasis sintomática, sin mejoría después de colecistectomía, y a quien, por medio de la colangiopancreatografía retrógrada endoscópica (CPRE) se le extrajo del colédoco un parásito vivo. Este fue confirmado, mediante patología, como Fasciola hepatica?

En algunos casos graves, la invasión crónica del parásito en la vía biliar provoca la obstrucción de esta y estasis biliar, ${ }^{4}$ lo que puede ocasionar desde una atrofia hepática con cirrosis periportal, colecistitis o colelitiasis, hasta complicaciones más severas como colangitis, abscesos hepáticos o pancreatitis aguda, poniendo de manifiesto en casos prolongados una colangitis esclerosante $y$ cirrosis biliar. $^{8}$

El diagnóstico de fascioliasis debe comprender las manifestaciones clínicas y los criterios epidemiológicos y laboratoriales. El estudio coproparasitológico se basa en el descubrimiento de huevos del parásito en las heces del paciente, tal como sucedió en nuestro caso. Es importante recalcar la utilidad de este estudio en la fase crónica.

La técnica Kato-Katz detecta la carga parasitaria mediante la aclaración de las heces y permite realizar el contaje de los huevos del parásito por gramo de heces. Esta técnica también sirve para realizar el seguimiento y evaluar la efectividad del tratamiento brindado. ${ }^{9-11}$

El diagnóstico serológico es por pruebas de inmunoensayo, detectando anticuerpos IgG específicos circulantes. Este método diagnóstico es utilizado en las fases aguda y crónica. Dentro de las pruebas, según se demostró en una revisión, la Cathepsin L - ELISA es la de mejor rendimiento en cuanto a sensibilidad y especificidad $(99,9 \%)$ para ambos indicadores, seguida de la Fas2 ELISA. ${ }^{10,12,13}$

La ecografía abdominal proporciona información acerca del compromiso hepático, la presencia de nódulos múltiples o lesiones irregulares y de masas quísticas y, en algunas ocasiones, permite observar los parásitos adultos en la vesícula biliar. La tomografía axial computarizada es útil en la fase aguda, en la que podemos observar hallazgos característicos, tales como nódulos hipodensos con tortuosidades o lineares que se dirigen al hilio hepático. ${ }^{14}$

La CPRE es un método diagnóstico y terapéutico para la obstrucción biliar por parasitosis. Permite el acceso a las vías biliares para así poder extraer el causal de la oclu- 
sión biliar. Esto se realiza a través de la esfinterotomía endoscópica seguida de extracción con balón o canastilla, siendo este el mejor método para tratar la infección parasitaria de vía biliar. Este procedimiento, además, debe acompañarse con el tratamiento antiparasitario. ${ }^{8,15}$ Dentro de los avances en el abordaje endoscópico, la utilidad del sistema de colangioscopía de un solo operador SpyGlass está asociada con un alto éxito del procedimiento, porque logra visualizar lo que por métodos endoscópicos convencionales no se podría, considerando que existen reportes de casos en los que se utilizó la técnica como método diagnóstico y terapéutico. ${ }^{16}$

En algunos casos de fascioliasis masiva o de resistencia al tratamiento oral, se ha considerado, según Villa-Gómez y Lazo Molina, el uso de la instilación transendoscópica de solución de povidona yodada al 2,5\%, en una cantidad de $20 \mathrm{ml}$ por $10 \mathrm{~min}$, para garantizar la muerte definitiva de los parásitos adultos y de los huevos presentes en el árbol biliar. ${ }^{8,17}$

El tratamiento antiparasitario de elección aceptado por la Organización Mundial de la Salud (OMS) es el triclabendazol $10 \mathrm{mg} / \mathrm{kg}$ en dosis única, ${ }^{18,19}$ que es efectivo contra las formas maduras e inmaduras del parásito; sin embargo, se han reportado casos de resistencia al fármaco cuando se administra en monodosis en nuestro país, tanto en animales como en seres humanos, ${ }^{20} \mathrm{y}$ algunos estudios no recomiendan la monodosis por el riesgo de aparición de cepas resistentes de Fasciola hepatica. ${ }^{10,21}$ Frente a ello, el Centro para el Control y Prevención de Enfermedades recomienda una doble dosis de triclabendazol $10 \mathrm{mg} / \mathrm{kg}$ cada $12 \mathrm{~h}$ y no aprueban el uso de otro medicamento alternativo en los humanos. ${ }^{22}$

Pese a ello, algunos estudios han descrito la efectividad de la nitaxozanida, ${ }^{9}$ incluso como terapia empleada tras el fallo de la terapia con triclabendazol. ${ }^{23}$

\section{Conclusión}

En el Perú, así como en el resto de América del Sur, la fascioliasis es una parasitosis endémica. El humano es uno de los huéspedes definitivos; se infecta al consumir agua o plantas acuáticas contaminadas con metacercarias.

El tratamiento de elección aprobado por la OMS es el triclabendazol $10 \mathrm{mg} / \mathrm{kg}$ en monodosis; no obstante, se han reportado casos de resistencia.

La CPRE es un método diagnóstico y terapéutico, porque permite visualizar la ubicación en el tracto biliar de formas adultas del parásito para su extracción y, además, es un método preventivo para el desarrollo de complicaciones como cirrosis biliar secundaria, colangitis y pancreatitis aguda.
Conflictos de interés. Este estudio no presentó ningún conflicto de interés entre los autores.

Consentimiento para la publicación. Para la confección de este manuscrito, se utilizaron datos anonimizados que no han distorsionado su significado cientifico.

Propiedad intelectual. Los autores declaran que los datos y las figuras presentes en el manuscrito son originales y fueron realizados en sus instituciones pertenecientes.

\section{Aviso de derechos de autor}

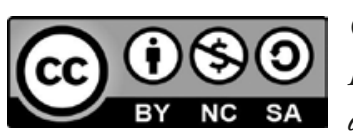

(C) 2021 Acta Gastroenterológica Latinoamericana. Este es un artículo de acceso abierto publicado bajo los términos de la Licencia Creative Commons Attribution (CC BY-NC-SA 4.0), la cual permite el uso, la distribución $y$ la reproducción de forma no comercial, siempre que se cite al autor y la fuente original.

Cite este artículo como: Geldres Molina AB, Alcántara Figueroa CE, Romero Garcia JL y col. Fascioliasis, una causa infrecuente de obstrucción de vía biliar: reporte de un caso. Acta Gastroenterol Latinoam. 2021;51(3): 347-52. https://doi.org/10.52787/werx8637

\section{Referencias}

1. Jarnagin WR. Blumgart's Surgery of the Liver, Pancreas and Biliary Tract E-Book. Elsevier Health Sciences. 2016:2120.

2. Marcos LA, Terashima A, Leguia G. La infección por Fasciola hepatica en el Perú: una enfermedad emergente. Rev gastroenterol Perú. 2007;27(4):389-96.

3. Müller I, Vásquez J, Sáenz R, Müller N, y cols. Obstrucción biliar por fascioliasis hepática. Gastroenterol latinoam. 2012;23(4):201-5.

4. De Jesús Herrera Esquivel J, Medina SR, Esquivel JCG. Ictericia obstructiva secundaria a presencia de Fasciola hepatica. Reporte de caso. Gastroenterología y Hepatología. 2011;34:435-7.

5. Kang BK, Jung B-K, Lee YS, Hwang IK, Lim H, Cho J, et al. A case of Fasciola hepatica infection mimicking cholangiocarcinoma and ITS-1 sequencing of the worm. Korean J Parasitol. 2014;52(2):193-6.

6. Vázquez-Elizondo G, Zavala-García C, Elizondo J. Infestación por Fasciola hepatica en la vía biliar. Médica. 2007;14(1).

7. Basile J, Branch SM, Desai SV, Arnold C, Smith A, Lee T-H. Hepatobiliary Fascioliasis: An Uncommon Cause of Biliary Obstruction in the United States. ACG Case Reports Journal. 2014;1:124-5.

8. Lazo Molina L, Garrido Acedo R, Cárdenas Ramírez B, Torreblanca Nava J. Extracción endoscópica por CPRE de Fasciola hepatica viva: reporte de dos casos y revisión de la literatura. Rev Gastroenterol Peru. 2013;33(1):75-81. 
9. Albán Olaya M, Jave Ortiz J, Quispe Lazo T. Fasciolasis en Cajamarca. Rev Gastroenterol Peru. 2002;22(1):28-32.

10. Resolución Ministerial No 266-2019/MINSA. Disponible en: https:/www.gob.pe/institucion/minsa/normas-legales/267535266-2019-minsa

11. Espinoza JR, Terashima A, Herrera-Velit P, Marcos LA. Fasciolosis humana y animal en el Perú: impacto en la economía de las zonas endémicas. Rev Peru Med Exp Salud Pública. 2010;27(4):604-12.

12. Millán AM, Wagenknecht SR, Cardenas PA, Carrasco LC. Common bile duct fasciolasis. Report of one case. Rev Chil Cir. 2008;60(4):332-5.

13. Muñoz Zambrano ME, Placencia Medina M, Del Pozo Muñoz JA, Sevilla Andrade C, Huiza Franco A. Diagnóstico serológico de la infección por Fasciola hepatica: una revisión sistemática. Revista de Gastroenterología del Perú. 2020;40(2):155-61.

14. González-Miguel J, Becerro-Recio D, Siles-Lucas M. Insights into Fasciola hepatica Juveniles: Crossing the Fasciolosis Rubicon. Trends Parasitol. 2021;37(1):35-47.

15. Martínez López R, Ruiz Torres J. Diagnóstico de la fasciolosis de las vías biliares por imagenología. Revista Cubana Med Trop. 2000;52(2):145-7.

16. Guzmán-Calderón E, Vera-Calderón A, Díaz-Ríos R, ArcanaLópez R, Alva-Alva E. Fasciola hepatica in the common bile duct: spyglass visualization and endoscopic extraction. Rev Esp Enferm Dig. 2018;110(10):671-3.
17. Villa-Gómez Roig G, Mahler MA, Manazzoni D, Villa-Gómez M, Vidales G, Marcolongo M. Spectrum of biliary parasites affecting the biliary tree (Fasciola hepatica, Echinococcus granulosus, and Ascaris lumbricoides). Endoscopy. 2018;50(8):E224-6.

18. Webb CM, Cabada MM. Recent developments in the epidemiology, diagnosis, and treatment of Fasciola infection. Curr Opin Infect Dis. 2018;31(5):409-14.

19. Gandhi P, Schmitt EK, Chen C-W, Samantray S, Venishetty VK, Hughes D. Triclabendazole in the treatment of human fascioliasis: a review. Transactions of The Royal Society of Tropical Medicine and Hygiene. 2019;113:797-804.

20. Ortiz P, Scarcella S, Cerna C, Rosales C, Cabrera M, Guzmán $\mathrm{M}$, et al. Resistance of Fasciola hepatica against Triclabendazole in cattle in Cajamarca (Peru): a clinical trial and an in vivo efficacy test in sheep. Vet Parasitol. 2013;195(1-2):118-21.

21. Terashima A, Marcos LA. Fracaso de dosis única de Triclabendazole para el tratamiento de fasciolosis humana. Acta Médica Perú. 2016;33(3):228.

22. Marcos L, Maco V, Terashima A. Triclabendazole for the treatment of human fascioliasis and the threat of treatment failures. Expert Rev Anti Infect Ther. 2020;1-7.

23. Ramadan HK-A, Hassan WA, Elossily NA, Ahmad AA, Mohamed AA, Abd-Elkader AS, et al. Evaluation of nitazoxanide treatment following triclabendazole failure in an outbreak of human fascioliasis in Upper Egypt. PLoS Negl Trop Dis. 2019;13(9):e0007779. 